\title{
ON THE NUMERICAL SOLUTION OF SINGULAR INTEGRAL EQUATIONS*
}

BY

\author{
F. ERDOGAN AND G. D. GUPTA
}

Lehigh University

\begin{abstract}
In this paper a pair of Gauss-Chebyshev integration formulas for singular integrals are developed. Using these formulas a simple numerical method for solving a system of singular integral equations is described. To demonstrate the effectiveness of the method, a numerical example is given. In order to have a basis of comparison, the example problem is solved also by using an alternate method.

1. Introduction. The solution of a large class of mixed boundary value problems in physics and engineering can be reduced to that of a system of singular integral equations of the following form:

$$
\begin{aligned}
\frac{1}{\pi} \int_{-1}^{1} \sum_{1}^{M} a_{i j} \phi_{j}(t) \frac{d t}{t-x}+\int_{-1}^{1} \sum_{1}^{M} k_{i j}(x, t) \phi_{j}(t) d t & =g_{i}(x), \\
-1 & <x<1, \quad i=1, \cdots, M .
\end{aligned}
$$
\end{abstract}

In (1.1) $\phi_{1}, \cdots, \phi_{M}$ are the unknown functions, the constant matrix $\left(a_{i j}\right)$ is nonsingular, the kernels $k_{i j}(x, t),(i, j=1, \cdots, M)$, are bounded in the closed domain $-1 \leq(x, t) \leq 1$, and $g_{1}, \cdots, g_{M}$ are the known input functions. In physical problems the function $\phi_{i}$ may be either a potential (e.g., temperature, displacement, velocity potential, electrostatic field) or a flux-type quantity (e.g., heat flux, stress, dislocation, velocity, charge density). The end points $\mp 1$ are points of geometric singularity. At these points $\phi$, is bounded if it is a potential and has an integrable singularity if it is a flux-type quantity.

The singular behavior of the functions $\phi_{j}(t)$ around $t=\mp 1$ may be obtained by analyzing the dominant part of the integral equations (1.1) through the use of functiontheoretic methods [1]. By multiplying (1.1) by $A^{-1}, A=\left(a_{i j}\right)$, and by following the procedure of [1], it can easily be shown that the fundamental functions of (1.1) which characterize the singular behavior of $\phi_{i}$ are given by

$$
R_{i}(t)=(1+t)^{(-1 / 2)+\alpha}(1-t)^{(1 / 2)+\beta}
$$

where $\alpha, \beta=0, \mp 1 ;-1<-\frac{1}{2}+\alpha<1,-1<\frac{1}{2}+\beta<1$ and the index of the integral equation is $\kappa=-(\alpha+\beta)=\mp 1$. Thus, if $\phi_{i}$ is bounded at the singular points, $\alpha=1$, $\beta=0$, and

$$
R_{i}(t)=\left(1-t^{2}\right)^{1 / 2}, \quad \kappa=-1 ;
$$

* Received February 5, 1971; revised version received May 27, 1971. This research was supported by National Science Foundation under Grant GK-11977. 
if $\phi_{;}$has an integrable singularity, $\alpha=0, \beta=-1$, and

$$
R_{j}(t)=\left(1-t^{2}\right)^{-1 / 2}, \quad \kappa=1 .
$$

For $\kappa=1$, the solution of (1.1) contains $M$ arbitrary constants which are usually determined by using the following set of (physical) conditions:

$$
\int_{-1}^{1} \phi_{i}(t) d t=C_{i}, \quad j=1, \cdots, M,
$$

where $C_{1}, \cdots, C_{M}$ are known constants.

The general theory of the system of singular integral equations (1.1) has been extensively studied (see, for example, [1] and [2]). The conventional method of solving (1.1) is based on the regularization of the system through the application of another singular operator (usually the adjoint of that of (1.1)). By expressing the unknown functions as

$$
\phi_{j}(t)=R_{i}(t) F_{i}(t)
$$

this method leads to a system of Fredholm integral equations in the new set of unknown functions $F_{j}(t)$ which are bounded and continuous in the interval $-1 \leq t \leq 1$. However, from the viewpoint of numerical analysis, the method is rather cumbersome and very laborious.

An effective approximate method preserving the correct nature of singularities of the functions $\phi_{i}$ is described in [3]. Here, noting that the fundamental functions $R_{i}(t)$ given by (1.3) or (1.4) are the weights of Chebyshev polynomials $U_{k}(t)$ or $T_{k}(t)$, and expressing the bounded functions $F_{i}$ defined by (1.6) as

$$
F_{j}(t)=\sum_{0}^{\infty} A_{j k} U_{k}(t) \quad \text { or } \quad F_{j}(t)=\sum_{0}^{\infty} B_{i k} T_{k}(t),
$$

the solution of (1.1) is reduced to that of an infinite system of linear algebraic equations in the unknown coefficients $A_{j k}$ or $B_{i k}(j=1, \cdots, M, k=0,1,2, \cdots)$ (see the example given in this paper).

In this paper we will describe a more direct numerical method of solving the system of singular integral equations (1.1). The method is based on the notion that by selecting the nodal points $t_{k}$ and $x_{k}$ in the interval $(-1,1)$ properly, the system $(1.1)$ can be treated as if it were a system of Fredholm equations and the unknown functions $F_{i}(t)$ may be determined by using the conventional collocation technique.

2. Two auxiliary formulas. Before describing the numerical method we will prove the validity of the following formulas:

$$
\begin{aligned}
\sum_{k=1}^{n} \frac{T_{j}\left(t_{k}\right)}{n\left(t_{k}-x_{r}\right)} & =0, \quad j=0 \\
& =U_{j-1}\left(x_{r}\right), \quad 0<j<n \\
T_{n}\left(t_{k}\right) & =0, \quad U_{n-1}\left(x_{r}\right)=0
\end{aligned}
$$

and

$$
\begin{gathered}
\sum_{k=1}^{n} \frac{\left(1-t_{k}^{2}\right) U_{j}\left(t_{k}\right)}{(n+1)\left(t_{k}-x_{\tau}\right)}=-T_{j+1}\left(x_{\tau}\right), \quad(n>j) \\
U_{n}\left(t_{k}\right)=0, \quad T_{n+1}\left(x_{\tau}\right)=0
\end{gathered}
$$


where $T_{n}(x)$ and $U_{n}(x)$ are Chebyshev polynomials defined by

$$
T_{n}(x)=\cos n \theta, U_{n}(x)=\frac{\sin (n+1) \theta}{\sin \theta}, \cos \theta=x .
$$

To prove (2.1) consider the following simple fraction expansion:

$$
\frac{U_{n-i-1}(x)}{T_{n}(x)}=\sum_{k=1}^{n} \frac{a_{k}}{t_{k}-x}, \quad T_{n}\left(t_{k}\right)=0
$$

where

$$
a_{k}=-\left(U_{n-i-1}\left(t_{k}\right)\right) /\left(T_{n}^{\prime}\left(t_{k}\right)\right)=-\left(U_{n-i-1}\left(t_{k}\right)\right) /\left(n U_{n-1}\left(t_{k}\right)\right) .
$$

Using the recursion formula [4]

$$
U_{n-i-1}(x)=T_{i}(x) U_{n-1}(x)-T_{n}(x) U_{i-1}(x)
$$

and noting that $T_{n}\left(t_{k}\right)=0$, from (2.4) and (2.5) we obtain

$$
\sum_{k=1}^{n} \frac{T_{j}\left(t_{k}\right)}{n\left(t_{k}-x\right)}=-\frac{U_{n-i-1}(x)}{T_{n}(x)} .
$$

For $x=x_{r}$ and $U_{n-1}\left(x_{r}\right)=0, j=0$ part of (2.1) follows from (2.7). For $j>0$ from (2.6) and (2.7) we obtain

$$
\sum_{k=1}^{n} \frac{T_{i}\left(t_{k}\right)}{n\left(t_{k}-x\right)}=U_{i-1}(x)-\frac{T_{i}(x) U_{n-1}(x)}{T_{n}(x)}
$$

which reduces to (2.1) for $x=x_{r}, U_{n-1}\left(x_{r}\right)=0$.

To prove (2.2) consider the following expansion:

$$
\frac{U_{n-i-1}(x)}{U_{n}(x)}=\sum_{1}^{n} \frac{b_{k}}{t_{k}-x}
$$

where, noting that $U_{n}\left(t_{k}\right)=0$ and [4]

$$
\left(1-t^{2}\right) U_{n}^{\prime}(t)=(n+1) U_{n-1}(t)-n t U_{n}(t),
$$

the constants $b_{k}$ are given by

$$
b_{k}=-\frac{U_{n-i-1}\left(i_{k}\right)}{U_{n}^{\prime}\left(t_{k}\right)}=-\frac{\left(1-t_{k}^{2}\right) U_{n-i-1}\left(t_{k}\right)}{(n+1) U_{n-1}\left(t_{k}\right)} .
$$

Using (2.6), the recursion formulas [4]

$$
T_{n}(t)=U_{n}(t)-t U_{n-1}(t), \quad U_{j}(t)=T_{i}(t)+t U_{i-1}(t)
$$

and again noting that $U_{n}\left(t_{k}\right)=0$, from (2.9) and (2.11) we obtain

$$
\sum_{k=1}^{n} \frac{\left(1-t_{k}^{2}\right) U_{j}\left(t_{k}\right)}{(n+1)\left(t_{k}-x\right)}=-\frac{U_{n-j-1}(x)}{U_{n}(x)}
$$

Using (2.6) now for $j \rightarrow j+1$ and $n \rightarrow n+1$, (2.13) becomes

$$
\sum_{k=1}^{n} \frac{\left(1-t_{k}^{2}\right) U_{i}\left(t_{k}\right)}{(n+1)\left(t_{k}-x\right)}=-T_{i+1}(x)+\frac{U_{j}(x) T_{n+1}(x)}{U_{n}(x)}
$$


which reduces to $(2.2)$ for $x=x_{r}, T_{n+1}\left(x_{r}\right)=0$.

3. Gauss-Chebyshev integration formulas for singular integrals. Consider now the following singular integral:

$$
S(x)=\frac{1}{\pi} \int_{-1}^{1} \frac{\phi(t) d t}{t-x}, \quad-1<x<1,
$$

where

$$
\phi(t)=R(t) F(t), \quad R(t)=\left(1-t^{2}\right)^{\mp 1 / 2}
$$

and $F(t)$ is bounded in $-1 \leq t \leq 1$.

(a) The case of $\kappa=1$. In this case $R(t)=\left(1-t^{2}\right)^{-1 / 2}$ is the weight of $T_{i}(t)$. Let us assume that in $-1 \leq t \leq 1$ the function $F(t)$ can be approximated to a sufficient degree of accuracy by the following truncated series:

$$
F(t) \simeq \sum_{0}^{p} B_{i} T_{i}(t)
$$

Thus (3.1) may be expressed as

$$
S(x) \simeq \sum_{i=0}^{D} B_{i} \frac{1}{\pi} \int_{-1}^{1} \frac{T_{i}(t) d t}{(t-x)\left(1-t^{2}\right)^{1 / 2}}=\sum_{i=1}^{D} B_{i} U_{i-1}(x)
$$

where the following relation is used [4]:

$$
\begin{aligned}
\frac{1}{\pi} \int_{-1}^{1} \frac{T_{j}^{\prime}(t) d t}{(t-x)\left(1-t^{2}\right)^{1 / 2}} & =0, \quad j=0 \\
& =U_{i-1}(x), \quad j>0, \quad(-1<x<1) .
\end{aligned}
$$

For $x=x_{r}$ it follows from (3.4), (3.3), and (2.1) that

$$
\begin{aligned}
S\left(x_{r}\right) & =\frac{1}{\pi} \int_{-1}^{1} \frac{F(t)}{t-x_{r}} \frac{d t}{\left(1-t^{2}\right)^{1 / 2}} \simeq \sum_{i=1}^{D} B_{i} U_{i-1}\left(x_{r}\right) \\
& =\sum_{j=1}^{\infty} \sum_{k=1}^{n} \frac{B_{i} T_{i}\left(t_{k}\right)}{n\left(t_{k}-x_{r}\right)}=\sum_{k=1}^{n} \frac{F\left(t_{k}\right)}{n\left(t_{k}-x_{r}\right)}
\end{aligned}
$$

where

$$
\begin{aligned}
T_{n}\left(t_{k}\right) & =0, t_{k}=\cos ((\pi / 2 n)(2 k-1)), \quad(k=1, \cdots, n) \\
U_{n-1}\left(x_{r}\right) & =0, x_{r}=\cos (\pi r / n), \quad(r=1, \cdots, n-1) .
\end{aligned}
$$

If one considers the Gauss-Chebyshev integration formula [5]

$$
\frac{1}{\pi} \int_{-1}^{1} \frac{f(t) d t}{\left(1-t^{2}\right)^{1 / 2}} \simeq \sum_{1}^{n} \frac{f\left(t_{k}\right)}{n}, \quad T_{n}\left(t_{k}\right)=0,
$$

it is seen that (3.6) formally represents the Gauss-Chebyshev integration formula for the singular integrals which is valid only at the discrete set of points $x=x_{\mathrm{r}}(r=$ $1, \cdots, n-1), U_{n-1}\left(x_{r}\right)=0$.

(b) The case of $k=-1$. For this case $R(t)=\left(1-t^{2}\right)^{1 / 2}$ is the weight of $U_{i}(t)$ and we will again assume that the following truncated series represents $F(t)$ with sufficient accuracy: 


$$
F(t) \simeq \sum_{0}^{D} A_{i} U_{j}(t)
$$

From the relation [4]

$$
\frac{1}{\pi} \int_{-1}^{1} \frac{U_{j}(t)\left(1-t^{2}\right)^{1 / 2}}{t-x} d t=-T_{i+1}(x), \quad(-1<x<1)
$$

and (3.1), (3.2) and (3.9), it follows that

$$
S(x) \simeq \sum_{i=0}^{p} A_{i} \frac{1}{\pi} \int_{-1}^{1} \frac{U_{j}(t)\left(1-t^{2}\right)^{1 / 2}}{t-x} d t=-\sum_{j=0}^{p} A_{i} T_{j+1}(x) .
$$

For $x=x_{r}$, using (2.2) and (3.9), (3.11) may be expressed as

$$
\begin{aligned}
S\left(x_{r}\right) & =\frac{1}{\pi} \int_{-1}^{1} \frac{F(t)}{t-x_{r}}\left(1-t^{2}\right)^{1 / 2} d t \simeq-\sum_{0}^{p} A_{i} T_{i+1}\left(x_{r}\right) \\
& =\sum_{i=0}^{p} \sum_{k=1}^{n} \frac{\left(1-t_{k}^{2}\right) A_{j} U_{i}\left(t_{k}\right)}{(n+1)\left(t_{k}-x_{r}\right)}=\sum_{k=1}^{n} \frac{\left(1-t_{k}^{2}\right) F\left(t_{k}\right)}{(n+1)\left(t_{k}-x_{r}\right)}
\end{aligned}
$$

where

$$
\begin{aligned}
U_{n}\left(t_{k}\right) & =0, t_{k}=\cos (k \pi /(n+1)), \quad(k=1, \cdots, n) \\
T_{n+1}\left(x_{r}\right) & =0, x_{r}=\cos (\pi(2 r-1) / 2(n+1)), \quad(r=1, \cdots, n+1) .
\end{aligned}
$$

Again, if we consider the related Gauss-Chebyshev integration formula [5]

$$
\frac{1}{\pi} \int_{-1}^{1} f(t)\left(1-t^{2}\right)^{1 / 2} d t \simeq \sum_{1}^{n} \frac{\left(1-t_{k}^{2}\right) f\left(t_{k}\right)}{n+1}, \quad U_{n}\left(t_{k}\right)=0
$$

it is seen that formally (3.12) may be considered as the Gauss-Chebyshev integration formula for singular integrals which is valid only at the discrete set of points $x=x_{r}$ $(r=1, \cdots, n+1), T_{n+1}\left(x_{r}\right)=0$.

Note 1: The only error in the formulas (3.6) and (3.12) comes from the truncated series representation of the function $F(t)$ by (3.3) and (3.9), indicating that, if $F$ is bounded and continuous, arbitrarily high accuracy can be obtained by choosing $n$ sufficiently large.

Note 2: Even though the number $p$ shown in (3.3) and (3.9) does not appear in the final equations (3.6) and (3.12), in deriving them it is assumed that $n>p$.

4. Solution of singular integral equation. Let us now consider the following singular integral equation:

$$
\frac{1}{\pi} \int_{-1}^{1} \frac{\phi(t)}{t-x} d t+\int_{-1}^{1} k(x, t) \phi(t) d t=g(x), \quad-1<x<1
$$

(a) The case of $k=1$. For this case the unknown function $\phi$ may be expressed as

$$
\phi(t)=F(t) /\left(1-t^{2}\right)^{1 / 2}
$$

where the bounded function $F$ is the new unknown. Substituting from (4.2) into (4.1), we obtain

$$
\frac{1}{\pi} \int_{-1}^{1} \frac{F(t) d t}{(t-x)\left(1-t^{2}\right)^{1 / 2}}+\int_{-1}^{1} k(x, t) \frac{F(t) d t}{\left(1-t^{2}\right)^{1 / 2}}=g(x) .
$$


$F(t)$ has to be obtained from (4.3) subject to the condition that (see (1.5))

$$
\int_{-1}^{1} \frac{F(t)}{\left(1-t^{2}\right)^{1 / 2}} d t=C
$$

where $C$ is a known constant. Note that since $F(t)$ and $k(x, t)$ are bounded, the second integral in (4.3) and the integral in (4.4) can be evaluated by using the Gauss-Chebyshev integration formula (3.8). Thus, from (3.6), (3.8), (4.3) and (4.4) we obtain

$$
\begin{gathered}
\sum_{k=1}^{n} \frac{1}{n} F\left(t_{k}\right)\left[\frac{1}{t_{k}-x_{r}}+\pi k\left(x_{r}, t_{k}\right)\right]=g\left(x_{r}\right) \quad(r=1, \cdots, n-1), \\
\sum_{1}^{n} \frac{\pi}{n} F\left(t_{k}\right)=C, \quad t_{k}=\cos \frac{\pi}{2 n}(2 k-1), \quad x_{r}=\cos \frac{\pi r}{n} .
\end{gathered}
$$

(4.5) provides a system of $n$ linear algebraic equations to determine $F\left(t_{1}\right), \cdots, F\left(t_{n}\right)$.

(b) The case of $\kappa=-1$. In this case $\phi$ is of the form

$$
\phi(t)=F(t)\left(1-t^{2}\right)^{1 / 2} .
$$

Following a procedure similar to that for case (a), we obtain from (3.12), (3.14), (4.1) and (4.6)

$$
\begin{gathered}
\sum_{k=1}^{n} \frac{1-t_{k}^{2}}{n+1} F\left(t_{k}\right)\left[\frac{1}{t_{k}-x_{r}}+\pi k\left(x_{r}, t_{k}\right)\right]=g\left(x_{r}\right) \\
t_{k}=\cos (k \pi /(n+1)), \quad x_{r}=\cos (\pi(2 r-1) / 2(n+1)) .
\end{gathered}
$$

(4.7) is valid for $r=1, \cdots, n+1$; that is, there are $(n+1)$ possible collocation points to determine the $n$ unknowns $F\left(t_{1}\right), \cdots, F\left(t_{n}\right)$. Thus it is sufficient to choose only $n$ of these points to determine $F\left(t_{k}\right)$. (In practice $n$ may be selected as an even integer and the point corresponding to $r=n / 2+1$ may be ignored.)

Note that the extension of the formulas (4.5) and (4.7), which are given for a single integral equation, to the system of singular integral equations such as (1.1) is straightforward.

5. Example. As an example consider the following integral equation:

$$
\frac{1}{\pi} \int_{-1}^{1} \frac{\phi(t)}{t-x} d t-\lambda \int_{-1}^{x} \phi(t) d t=P_{0}, \quad-1<x<1
$$

subject to

$$
\int_{-1}^{1} \phi(t) d t=0
$$

Equation (5.1) represents the formulation of the plane elasticity problem for a cover plate bonded to an elastic half-space $y<0,-\infty<x<\infty$ along $y=0,-1<x<1$. The elastic constants of the plate and the half-space are $\mu_{1}, \kappa_{1}$ and $\mu_{2}, \kappa_{2}$ respectively. $\phi(t)(-1<t<1)$ is the shear stress acting on the interface. The thickness $h$ of the plate is sufficiently small to justify the assumption that $\sigma_{\nu} \simeq 0$ throughout the plate. The constant $\lambda$ is given by

$$
\lambda=\left(\left(1+\kappa_{1}\right) /\left(1+\kappa_{2}\right)\right)\left(\left(\mu_{2}\right) /\left(2 \mu_{1} h\right)\right) .
$$

The constant $P_{0}$ represents the external load; in this case, $P_{0}=-\sigma_{0} / 2$, where $\sigma_{0}=\sigma_{2 x}^{\infty}$ 
is the uniform stress acting on the half-space away from and parallel to the cover plate.

In this problem the shear stress $\phi(t)$ has integrable singularities at $t=\mp 1$. Thus $\phi$ may be expressed as

$$
\phi(t)=F(t) /\left(1-t^{2}\right)^{1 / 2}
$$

where $F(t)$ is a bounded function in the closed interval $-1 \leq t \leq 1$. From a practical viewpoint an important quantity to evaluate is the strength of the stress singularity at the end points $t=\mp 1$ which may be obtained from (5.4) as

$$
A=\lim _{t \rightarrow 1}\left(1-t^{2}\right)^{1 / 2} \phi(t)=F(1) .
$$

The function $F$ is obtained from (4.5). Here we will only give the results for the strength of the stress singularity $A$. For $\lambda=0$ (i.e., the inextensible cover plate), it is seen from (3.5) that the exact solution is $F(t)=P_{0} T_{1}(t)=P_{0} t$ and $A=P_{0}$. For $P_{0}=1, \lambda=\frac{10}{3}$ and $\lambda=\frac{1}{3}$, and for $n=20,40,60$ the calculated results are shown in Table $I$. The values of $A$ given in Table I are obtained from a quadratic extrapolation of $F\left(t_{k}\right)$ based on the last three points. The last column in the table is obtained from the solution of the problem by using the method given in [3]. This solution will now be described briefly.

Noting that $F(t)$ is an odd function, we let

$$
F(t)=\sum_{1}^{\infty} b_{i} T_{2 j-1}(t)
$$

and substitute into (5.1). Using (3.5) and performing the integrals, we find

$$
\sum_{1}^{\infty} b_{j}\left\{U_{2 j-2}(x)+\frac{\lambda}{2 j-1} \sin \left[(2 j-1) \cos ^{-1} x\right]\right\}=P_{0}, \quad-1<x<1 .
$$

An appropriate method of determining $b_{i}$ would be the reduction of (5.7) to an infinite algebraic system through a weighted residual technique with weight functions $U_{2 k}(x)$ $\left(1-x^{2}\right)^{1 / 2}(k=0,1,2, \cdots)$. Using the orthogonality relations

$$
\begin{aligned}
\frac{1}{\pi} \int_{-1}^{1} U_{j}(x) U_{k}(x)\left(1-x^{2}\right)^{1 / 2} d x & =0, & j \neq k \\
& =\frac{1}{2}, & j=k,
\end{aligned}
$$

we may then express (5.7) as

$$
\begin{aligned}
b_{k} & =\sum_{j=1}^{\infty} c_{k j} b_{j}+D_{k-1} \quad(k=1,2, \cdots), \\
c_{k i} & =\frac{2 \lambda}{\pi}\left(\frac{1}{(2 k-2)^{2}-(2 j-1)^{2}}-\frac{1}{(2 k)^{2}-(2 j-1)^{2}}\right), \\
D_{0} & =P_{0} ; \quad D_{k-1}=0, \quad k>1 .
\end{aligned}
$$

TABLE I

The strength of stress singularity, $F(1)$

\begin{tabular}{ccccc}
\hline$n$ & 20 & 40 & 60 & $A(30)$ \\
$10 / 3$ & 0.4061 & 0.4104 & 0.4115 & 0.4115 \\
$1 / 3$ & 0.8323 & 0.8331 & 0.8340 & 0.8334 \\
\hline
\end{tabular}


The infinite system (5.9) can be solved by using the method of reduction if it is regular or quasi-regular [6]. For regularity the coefficients must satisfy the following relation [6]:

$$
\sum_{i=1}^{\infty}\left|c_{k j}\right|<1 \quad(k=1,2, \cdots)
$$

From (5.9) and (5.10), after performing the sum, we obtain

$\frac{2 \lambda}{\pi}\left[\frac{8(2 k-1)}{(4 k-3)(4 k-1)}+2 \sum_{j=1}^{k-2}\left(\frac{1}{(2 k-2)^{2}-(2 j-1)^{2}}-\frac{1}{(2 k)^{2}-(2 j-1)^{2}}\right)\right]<1$.

In (5.11) the quantity in brackets takes its greatest value for $k=1$. Hence the range of $\lambda$ for which the system is regular is found to be

$$
\lambda<3 \pi / 16 \text {. }
$$

For values of $\lambda$ greater than $3 \pi / 16,(5.9)$ is quasi-regular in the sense that there exists a $k$ for which the infinite system beginning with the $k+1$ st equation is regular. The integral equation (5.1) has also been reduced to an infinite system in [7]. However, the procedure followed in [7] is extremely complicated and no examples are given.

From (5.5) and (5.6) the strength of the stress singularity may be expressed as

$$
A=A(\infty)=F(1)=\sum_{1}^{\infty} b_{k}, \quad A(N)=\sum_{1}^{x} b_{k} .
$$

(5.9) is solved by the method of reduction; that is, by considering only the first $N$ equations and $N$ unknowns. The calculated values of $A(N)$ are shown in Table II for $N=$ $5,10, \cdots, 30$ and $\lambda=\frac{10}{3}$ and $\lambda=\frac{1}{3}$. Note that

$$
\frac{10}{3}>\lambda_{c r}=\frac{3 \pi}{16}>\frac{1}{3}
$$

which is reflected in the convergence of $A(N)$ for the two cases. Nevertheless, the table shows that the convergence is very good for both cases.

In order to compare the results obtained by the two methods, the values of $F(x)$ calculated at a selected set of points $x$ are shown in Table III. The agreement between two sets of results seems to be quite satisfactory.

Regarding the convergence of the calculated results in the numerical work where it is conjectured that "exact" results would be obtained if one lets " $N \rightarrow \infty$ ", one may

TABLE II

The values of $A(N)$

\begin{tabular}{cc}
$\lambda=10 / 3$ & $\lambda=\frac{1}{3}$ \\
$\mathrm{~A}(5)=0.40245222$ & 0.83161663 \\
$\mathrm{~A}(10)=0.40955555$ & 0.83297383 \\
$\mathrm{~A}(15)=0.41081248$ & 0.83322133 \\
$\mathrm{~A}(20)=0.41124571$ & 0.83330764 \\
$\mathrm{~A}(25)=0.41144482$ & 0.83334753 \\
$\mathrm{~A}(30)=0.41155222$ & 0.83336917 \\
\hline
\end{tabular}


TABLE III

Comparison of $F(x), \lambda=\frac{1}{3}$

\begin{tabular}{ccc}
\hline & $F(x)$ & $F(x)$ \\
.11753740 & Eq. $(4.5), n=40$ & Eq. $(5.6), N=20$ \\
.27144045 & .08015834 & .08016911 \\
.41865974 & .18664307 & .18666094 \\
.55557023 & .29205415 & .29207916 \\
.67880075 & .39540625 & .39543827 \\
.78531693 & .49522078 & .49525949 \\
.87249601 & .58935251 & .58939725 \\
.93819134 & .67482044 & .67487044 \\
.98078528 & .74762613 & .74768109 \\
.99922904 & .80246307 & .80252529 \\
\hline
\end{tabular}

try to estimate the limiting values of the calculated results by suitably expressing them as functions of $1 / N$ and letting $N \rightarrow \infty$. For example, in the problem under consideration it may be assumed that for "large" values of $N$ the strength of the stress singularity $A(N)$, which is given in Table II, can be expressed as

$$
A(N)=A(\infty)+B / N^{\alpha}
$$

where $A(\infty), B$, and $\alpha$ are unknown constants and $A(\infty)$ is the desired quantity. These unknowns can be determined by writing (5.14) for $N=N_{i}, i=1,2,3$. As an example, the results obtained from Table II (which contain only a part of $A(N)$ ) for $\lambda=\frac{10}{3}$ are given in Table IV. The table shows the estimated value of $A(\infty)$ for various sets of $N_{\text {i }}$ $(i=1,2,3)$. Comparison of these values with each other and with those given in Table I needs no further elaboration.

From examination of (5.14) it may be seen that for stability of the calculated results, at $N=\infty d A / d(1 / N)$ should be zero; that is, $\alpha$ should be greater than one. Generally speaking, the greater the exponent $\alpha$, the higher the stability. If $\alpha<1$ the extrapolation may give very erroneous results. Also, if the behavior of $A\left(N_{i}\right)$ is oscillating in nature, the model given by (5.14) requires modification.

Conclusion. Based on the results given in this paper, and particularly those obtained for much more complicated problems which were worked out recently, the technique described in this paper seems to be an extremely simple and a very effective method for solving a system of singular integral equations of the first kind.

TABLE IV

Values of $A(\infty), B, \alpha(E q .(5.14), \lambda=10 / 3)$

\begin{tabular}{cccc}
\hline$N_{i}$ & $A(\infty)$ & $B$ & $\alpha$ \\
$6,8,10$ & 0.41181 & -0.25477 & 2.0544 \\
$8,12,16$ & 0.41180 & -0.24433 & 2.0358 \\
$10,11,12$ & 0.41187 & -0.21117 & 1.9588 \\
$10,20,30$ & 0.41179 & -0.23717 & 2.0246 \\
$28,29,30$ & 0.41179 & -0.22060 & 2.0018 \\
\hline
\end{tabular}




\section{REFERENCES}

[1] N. I. Muskhelishvili, Singular integral equations, P. Noordhoff Ltd., Groningen, Holland, 1953

[2] F. D. Gakhov, Boundary value problems, Pergamon Press, Oxford, 1966

[3] F. Erdogan, Approximate solutions of systems of singular integral equations, SIAM J. Appl. Math. 17, 1041-1059 (1969)

[4] A. Erdelyi (ed.), Higher transcendental functions, Vol. 2, McGraw-Hill, New York, 1953

[5] M. Abramowitz and I. A. Stegun, eds., Handbook of mathematical functions, National Bureau of Standards, Appl. Math. Series 55, 1964

[6] L. V. Kantorovich and V. I. Krylov, Approximate methods of higher analysis, Interscience, New York, 1958

[7] N. Kh. Arutiunian, Contact problem for a half-plane with elastic reinforcement, J. Appl. Math. Mech. (Transl. PMM) 32, 632-646 (1968) 